\title{
De Rham's theorem in a smooth topos
}

\author{
BY IEKE MOERDIJK AND GONZALO E. REYES \\ Universiteit van Amsterdam and Université de Montréal
}

(Received 25 January 1984)

It has been persuasively argued (e.g. by Law vere[8]) that the mathematical world picture needed to develop the physics of continuous bodies and fields should involve a cartesian closed category $\mathscr{E}$ of smooth morphisms between smooth spaces. As far as the foundations of the calculus of variations are concerned, the need for such a category was recognized by $K$. T. Chen (cf. [2]).

Since the usual category $\mathscr{M}$ of smooth manifolds is not cartesian closed $\left(C^{\infty}(M, N)\right.$ is not necessarily a manifold if $M$ and $N$ are), several cartesian closed extensions of $\mathscr{M}$ have been considered in the literature (see, for example, the forthcoming Proceedings of the Buffalo Conference on 'Foundations of Categories and Continuum Mechanics').

This paper deals with cohomological properties of manifolds viewed as objects, not of $\mathscr{H}$, but of a much larger category $\mathscr{G}$ of smooth spaces introduced by Dubuc [3] and further studied by Moerdijk and Reyes[10]. Not only is $\mathscr{G}$ such a cartesian closed extension of $\mathscr{M}$, but it moreover has the much richer closure properties of a Grothendieck topos. These closure properties allow us to perform the usual set-theoretic constructions of new objects from given ones within the topos $\mathscr{G}$ (for example, if $M$ and $N$ are objects of $\mathscr{G}$, then there is a function-space object $N^{M}$ in $\mathscr{G}$, and we can construct objects of the form $\{x \in M \mid \phi(x)\}$, or $\{S \subseteq M \mid \psi(S)\}$, etc.). Moreover, the usual set-theoretic arguments remain valid, provided they are constructive (equivalently, intuitionistic logic is used).

The consequences of this fact about $\mathscr{G}$ (and more generally about any Grothendieck topos) are obvious: the rather complex mathematical structure of the topos can be kept away from the language, which is now used to describe what goes on 'inside' the topos, rather than to talk about the topos from an 'external', classical point of view.

Furthermore, this 'constructive' set-theoretical reasoning within a topos opens the way to regard the topos as a model of a theory which may be inconsistent with classical logic. In our example, $\mathscr{G}$ is a model for Synthetic Differential Geometry (SDG for short; for general information, see Kock [6]).

Of course, the problem that arises immediately is whether the usual properties of manifolds can be extended to $\mathscr{G}$. As a particular case of this problem, we give in this paper extensions of several versions of De Rham's theorem to the topos $\mathscr{G}$, and compare these with their classical counterparts. As an application of this comparison to 'classical' analysis, we conclude that these versions of De Rham's theorem hold 'smoothly in parameters'.

\section{De Rham's theorem in $\mathscr{G}$}

We first recall the definition of the topos $\mathscr{G}$ and some of its basic properties. For details, the reader is referred to Moerdijk \& Reyes [10]. 
The topos $\mathscr{G}$ is the category of sheaves over a site $\mathbb{G}$. The underlying category of $\mathbb{G}$ is the opposite category of a category of rings, whose objects are the rings of the form

$$
C^{\infty}\left(\mathbb{R}^{n}\right) / I \text {. }
$$

where $C^{\infty}\left(\mathbb{R}^{n}\right)$ is the ring of smooth functions $\mathbb{R}^{n} \rightarrow \mathbb{R}$, and $I$ is a germ-determined ideal (i.e. $f \in I$ if for all $p \in \mathbb{R}^{n},\left.f\right|_{p}$, the germ of $f$ at $p$, is an element of the ideal $\left.I\right|_{p}$ of germs at $p$ of functions in $I$ ). We write $\bar{A}$ for the object of $\mathbb{G}$ corresponding to such a ring $A=C^{\infty}\left(\mathbb{R}^{n}\right) / I$, i.e. $\bar{A}$ is the (formal) dual of $A$. If $\bar{A}=\overline{C^{\infty}\left(\mathbb{R}^{n}\right) / I}$ and $\bar{B}=\overline{C^{\infty}\left(\mathbb{R}^{m}\right) / J}$ are objects of $\mathbb{G}$, a map $\bar{B} \rightarrow \bar{A}$ of $\mathbb{G}$ is by definition a ring homomorphism $A \rightarrow B$ which is induced by composition with a smooth function $\mathbb{R}^{n} \rightarrow \mathbb{R}^{m}$. Explicitly, a map $\bar{B} \rightarrow \bar{A}$ of $\mathbb{G}$ is an equivalence class of smooth functions $\mathbb{R}^{n} \stackrel{\phi}{\rightarrow} \mathbb{R}^{m}$ having the property that, for all smooth $\mathbb{R}^{n} \stackrel{f}{\rightarrow} \mathbb{R}$,

$$
f \in J \Rightarrow f \circ \phi \in I,
$$

and two such functions $\phi$ and $\phi^{\prime}$ are equivalent if for all the projections $\pi_{i}: \mathbb{R}^{m} \rightarrow \mathbb{R}$ $(i=1, \ldots, m), \pi_{i} \circ \phi=\pi_{i} \circ \phi^{\prime} \bmod I$.

In fact, $\mathbb{G}$ contains (isomorphic copies of) all duals of rings of the form $C^{\infty}(U) / I$, where $U$ is an open subspace of some euclidean space $\mathbb{R}^{n}$, and $I$ is a germ-determined ideal, and thus we can equip $\mathbb{G}$ with the Grothendieck topology generated by coverings of the form

$$
\overline{\left\{C^{\infty}\left(U_{\alpha}\right)\right.} \rightarrow \overline{\left.C^{\infty}\left(\mathbb{P}^{n}\right)\right\}_{\alpha}},
$$

where $\left\{U_{\alpha}\right\}_{\alpha}$ is an open cover of $\mathbb{R}^{n}$ and the maps come from the inclusions $U_{\alpha} \hookrightarrow \mathbb{R}^{n}$.

The point about these rings is that they are $C^{\infty}$-rings, meaning that all $C^{\infty}$-maps $\mathbb{R}^{n} \rightarrow \mathbb{R}$ are interpretable as operations on them and all equations between these in $\mathbb{R}$ also hold in these rings.

The site $G$ contains (copies of) all $\sigma$-compact manifolds, as a consequence of Whitney's embedding theorem. Hence by the Yoneda embedding $Y$ we have a copy $s(M)$ in $\mathscr{G}$ of every such manifold, and in fact $s$ is a full and faithful functor. Thus we have a diagram of categories and functors

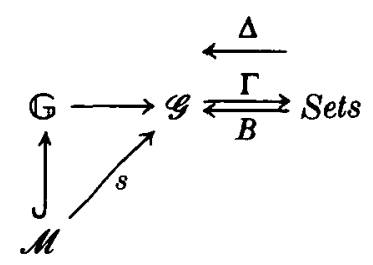

where $\Gamma$ is the global sections functor, $\Delta$ is the constant sheaf functor, and $B$ is the right adjoint of $\Gamma$,

$$
\Delta \dashv \Gamma \dashv B .
$$

In particular, in $\mathscr{G}$ we have 'smooth spaces' like

$$
\begin{aligned}
1 & =s\left(\mathbb{R}^{0}\right) & & \text { the point } \\
R & =s(\mathbb{R}) & & \text { the smooth line } \\
{[0,1] } & =s([0,1]) & & \text { the smooth unit interval. }
\end{aligned}
$$

As stated in the introduction, we can now perform the usual set-theoretical con- 
structions in $\mathscr{G}$, and, in particular, we can do differential topology in $\mathscr{G}$. Now differential topology in $\mathscr{G}$ is quite different from differential topology in Sets: in fact, and this is why $\mathscr{G}$ is so adequate for our purposes, $\mathscr{G}$ is a model of SDG. On the one hand, this means that we have our hands tied down to constructive arguments, but on the other hand we have a lot of useful structure available that degenerates in the classical context. For example, the subobject $D$ of $R$ defined by

$$
D=\left\{h \in R \mid h^{2}=0\right\},
$$

the set of 'first-order infinitesimals', is rather different in $\mathscr{G}$ from the one-point space, as illustrated by the fact that functions from $D$ correspond to tangent vectors: for every manifold $M \in \mathscr{M}$ we have a canonical isomorphism

$$
s(T M) \cong s(M)^{D} .
$$

In particular, the 'Kock-Lawvere axiom' $R \times R \stackrel{\sim}{\rightarrow} R^{D}$ holds in $\mathscr{G}$.

In classical differential topology, the De Rham complex of a manifold is built up from differential forms and exterior differentiation. In the context of SDG, these building blocks can be defined for any object $M$, since all objects are 'smooth spaces'. To define these notions (cf. Kock, Reyes and Veit[7]), let $M$ be any smooth space. An infinitesimal n-cube on $M$ is an element of $M^{D^{n}} \times D^{n}$, i.e. an $(n+1)$-tuple $\left(\gamma, h_{1}, \ldots, h_{n}\right)$. The object of infinitesimal $n$-chains, $C_{n}(M)$, is the free $R$-module generated by the infinitesimal $n$-cubes on $M$. So an element of $C_{n}(M)$ is a formal linear combination

$$
\sum_{i=1}^{\rho} a_{i}\left(\gamma_{i}, h_{1}^{i}, \ldots, h_{n}^{i}\right)
$$

where $a_{i} \in R,\left(\gamma_{i}, h_{1}^{i}, \ldots, h_{n}^{i}\right) \in M^{D^{n}} \times D^{n}$. An $n$-form on $M$ is a map

$$
\begin{gathered}
M^{D^{n}} \times D^{n} \stackrel{\omega}{\rightarrow} R \\
\left(\gamma, h_{1}, \ldots, h_{n}\right) \mapsto{ }_{\left(\gamma, h_{1}, \ldots, h_{n}\right)} \omega
\end{gathered}
$$

assigning an element of $R$ (like area, volume, etc.) to every infinitesimal $n$-cube, subject to the following three conditions:

(1) Homogeneity. $\omega\left(a_{i} \cdot \gamma, h_{1}, \ldots, h_{n}\right)=a \cdot \omega\left(\gamma, h_{1}, \ldots, h_{n}\right)$, where $a_{i} \cdot \gamma: D^{n} \rightarrow M$ is defined by

$$
a_{i} \cdot \gamma\left(x_{1}, \ldots, x_{i}, \ldots, x_{n}\right)=\gamma\left(x_{1}, \ldots, a \cdot x_{i}, \ldots, x_{n}\right),
$$

for every $a \in R$ and every infinitesimal $n$-cube $\left(\gamma_{1}, h_{1}, \ldots, h_{n}\right)$.

(2) Alternance. $\omega\left(\sigma \gamma, h_{1}, \ldots, h_{n}\right)=\operatorname{sgn}(\sigma) \cdot \omega\left(\gamma, h_{\sigma(1)}, \ldots, h_{\sigma(n)}\right)$, where $\sigma$ is any permutation of $\{1, \ldots, n\}$, and $\sigma \gamma$ is $\gamma$ composed with the coordinate permutation induced by $\sigma$, i.e.

$$
\sigma \gamma\left(x_{1}, \ldots, x_{n}\right)=\gamma\left(x_{\sigma(1)}, \ldots, x_{\sigma(n)}\right)
$$

$\operatorname{sgn}(\sigma)$ is the signature of $\sigma$.

(3) Degeneracy. $\omega\left(\gamma, h_{1}, \ldots, 0, \ldots, h_{n}\right)=0$.

The object of $n$-forms on $M$ is denoted by $\Lambda^{n}(M)$.

Note that, by the Kock-Lawvere axiom $R^{D} \cong R \times R$ and the degeneracy condition, each $n$-form $\omega$ on $M$ can be written as

$$
\omega\left(\gamma, h_{1}, \ldots, h_{n}\right)=h_{1} \cdot \ldots \cdot h_{n} \cdot \tilde{\omega}(\gamma)
$$


for a unique $\tilde{\omega}: M^{D^{n}} \rightarrow R$. This map $\tilde{\omega}$ satisfies the homogeneity condition $\left(\tilde{\omega}\left(a_{i} \cdot \gamma\right)=\right.$ $a \cdot \tilde{\omega}(\gamma))$ and is alternating $(\tilde{\omega}(\sigma \gamma)=\operatorname{sgn}(\sigma) \cdot \tilde{\omega}(\gamma))$. Thus, we obtain a 1-1 correspondence between elements $\omega \in \Lambda^{n}(M)$ and alternating homogeneous maps $\tilde{\omega}: M^{L^{n}} \rightarrow R$, and we will often identify the two.

If $\omega: M^{D^{n}} \times D^{n} \rightarrow R$ is an $n$-form on $M$, we will write

$$
\int_{(-)} \omega: C_{n}(M) \rightarrow R
$$

for the unique $R$-linear map extending $\omega$.

By taking the boundary of an infinitesimal $n$-cube we may define an $R$-linear boundary operator

$$
\partial: C_{n+1}(M) \rightarrow C_{n}(M)
$$

via $\partial\left(\gamma, h_{1}, \ldots, h_{n+1}\right)=\sum_{i=0}^{n+1} \sum_{\alpha=0,1}(-1)^{i+\alpha} F_{i \alpha}\left(\gamma, h_{1}, \ldots, h_{n+1}\right)$, where $F_{i \alpha}\left(\gamma, h_{1}, \ldots, h_{n+1}\right)$ is the infinitesimal $n$-cube

$$
\left(\left[\left(x_{1}, \ldots, x_{n}\right) \mapsto \gamma\left(x_{1}, \ldots, x_{i-1}, \alpha \cdot h_{i}, x_{i}, \ldots, x_{n}\right)\right], h_{1}, \ldots, \hat{h}_{i}, \ldots, h_{n+1}\right) .
$$

This operator $\partial$ enables us to define an $R$-linear map $\Lambda^{n}(M) \stackrel{d}{\rightarrow} \Lambda^{n+1}(M)$, the exterior differentiation map, by postulating the validity of the following infinitesimal version of Stokes' theorem:

$$
\int_{\left(\gamma, h_{1}, \ldots, h_{n}\right)} d \omega=\int_{\partial\left(\gamma, h_{1} \ldots, h_{n}\right)} \omega
$$

The De Rham complex of $R$-modules (and $R$-linear maps) of $M$ is the sequence

$$
\ldots \rightarrow \Lambda^{n-1}(M) \stackrel{d}{\rightarrow} \Lambda^{n}(M) \stackrel{d}{\rightarrow} \Lambda^{n+1}(M) \rightarrow \ldots
$$

where $\Lambda^{n}(M)=0$ for all $n<0$. The De Rham cohomology $R$-modules of $M$ are defined, as in the classical case, by

where

$$
H^{n}(M)=F^{n}(M) / E^{n}(M),
$$

and

$$
F^{n}(M)=\operatorname{Ker}\left(\Lambda^{n}(M) \stackrel{d}{\rightarrow} \Lambda^{n+1}(M)\right) \quad(\text { the } \text { closed } n \text {-forms) }
$$

$$
E^{n}(M)=\operatorname{Im}\left(\Lambda^{n-1}(M) \stackrel{d}{\rightarrow} \Lambda^{n}(M)\right) \quad \text { (the exact } n \text {-forms). }
$$

To state De Rham's theorem, we need the following version of the integration axiom:

$$
H^{1}([0,1])=(0),
$$

which holds in $\mathscr{G}$ (see Quê-Reyes[12]). Using this axiom, we can define integration of a form $\omega \in \Lambda^{n}(M)$ along an $n$-simplex $\gamma: \Delta_{n} \rightarrow M$ in the standard way.

We define the object $S_{n}(M)$ of singular $n$-chains of $M$, the boundary operator $\partial=\partial_{n}: S_{n}(M) \rightarrow S_{n-1}(M)$, the objects $Z_{n}(M)$ and $B_{n}(M)$ of $n$-cycles and $n$-boundaries of $M$, and the $n$-dimensional singular homology $R$-module of $M$,

$$
H_{n}(M ; R)=Z_{n}(M) / B_{n}(M),
$$

completely parallel to the classical definitions (the synthetic approach does not improve the usual presentation). 
After defining $\int_{\gamma} \omega$ for $\omega \in \Lambda^{n}(M)$ and generators $\gamma \in M^{\Delta_{n}}$ of $S_{n}(M)$, we extend this to a map

$$
\begin{aligned}
\Lambda^{n}(M) \times S_{n}(M) & \stackrel{\jmath}{\rightarrow} R \\
(\omega, \gamma) & \mapsto \int_{\gamma} \omega,
\end{aligned}
$$

which is $R$-linear in both $\omega$ and $\gamma$ separately. By the finite version of Stokes' theorem,

$$
\int_{\gamma} d \omega=\int_{\partial \gamma} \omega
$$

(as opposed to the infinitesimal version, which holds by definition; for a proof of the finite version, see Kock, Reyes and Veit[7]), the map ( passes to quotients, and we obtain an $R$-linear map

$$
I: H^{q}(M) \rightarrow H_{q}(M ; R)^{*}=\operatorname{Hom}_{R}\left(H^{q}(M ; R), R\right) .
$$

Our aim now is to perform, within $\mathscr{G}$, a Milnor-type proof of the classical version of De Rham's theorem stating that $I$ is an isomorphism for every object of the form $s(M)$, with $M \in \mathscr{M}$. We recall that this proof proceeds by checking that $H^{q}(s(M))$ and $\left(H_{q}(s(M) ; R)\right)^{*}$ satisfy the three axioms for a cohomology theory: the Poincaré lemma, the Mayer-Vietoris sequence, and additivity (cf., e.g. Bott and Tu[1]). To this end, it is necessary to check that $s$ preserves the ingredients of such a proof. And indeed, we can show:

Lemma. The canonical embedding $s: \mathscr{M} \hookrightarrow \mathscr{G}$ preserves partitions of unity, products, finite intersections of open subspaces, open covers, compactness and connectedness.

Preservation of products, finite intersections of opens, and open covers was already proved by Dubuc[3] (see also Moerdijk and Reyes [10]). The others were proved in [10]. (The case of partitions of unity was proved only for $R=s(\mathbb{R})$, and compactness only for $[0,1]=s[0,1]$, but exactly the same arguments will yield the corresponding results for any paracompact, resp. compact manifold.)

Using set-theoretic language in $\mathscr{G}$ (not sheaf-language!), the usual classical argument, which is constructive and explicit, gives

Theorem (De Rham's theorem in $\mathscr{G}$ ). For any $M \in \mathscr{M}$, the canonical integration map

is an $R$-linear isomorphism in $\mathscr{G}$.

$$
\begin{aligned}
I: H^{q}(s M) & \rightarrow H_{q}(s M ; R)^{*} \\
{[\omega] } & \mapsto\left([\gamma] \mapsto \int_{\gamma} \omega\right)
\end{aligned}
$$

And, just because the argument is completely parallel to the classical one,

THEOREM (comparison theorem for $\mathscr{G}$ ). For any manifold $M \in \mathscr{M}$ and any set $S$,

$$
\begin{aligned}
H_{q}(M ; \mathbb{R}) & \cong \oplus_{S} \mathbb{R} \quad \text { in Sets } \quad \text { iff } \quad H_{q}(s M ; R) \cong \oplus_{\Delta(S)} R \quad \text { in } \mathscr{G} \\
H^{q}(M) & \cong \Pi_{S} \mathbb{R} \quad \text { in Sets } \quad \text { iff } \quad H^{q}(s M) \cong \Pi_{\Delta(S)} R \quad \text { in } \mathscr{G},
\end{aligned}
$$

where the isomorphisms on the left are the global sections of those on the right.

(The skeptical reader may find details in Moerdijk and Reyes [11].) 


\section{Other cohomologies}

Now that we have established a form of De Rham's theorem in $\mathscr{G}$, it is natural to ask whether this theorem also holds for other cohomologies. We shall consider two examples of this question: the case of Cech cohomology and the case of singular cohomology.

We quickly recall the classical version of De Rham's theorem for Cech cohomology. Let $M \in \mathscr{M}$ be a manifold and let $\mathscr{U}=\left\{U_{\alpha}\right\}_{\alpha}$ be a good cover of $M$, i.e. an open cover such that all nonempty intersections $U_{\alpha_{0}} \cap \ldots \cap U_{\alpha_{n}}$ are diffeomorphic to some $\mathbb{R}^{k}$. Assume that the index set $\{\alpha\}$ is linearly ordered. The $\nearrow_{\text {ech }}$ complex (with coefficients in $\mathbb{R}$ ) is the complex

$$
C^{0}(\mathscr{U}, \mathbb{R}) \stackrel{\delta}{\rightarrow} C^{1}(\mathscr{U}, \mathbb{R}) \stackrel{\delta}{\rightarrow} C^{2}(\mathscr{U}, \mathbb{R}) \rightarrow \ldots,
$$

where $C^{n}(\mathscr{U}, \mathbb{R})$ is the vectorspace ( $\mathbb{R}$-module)

$$
\prod_{\alpha_{0}<\ldots<\alpha_{n}} F^{0}\left(U_{\alpha_{0} \ldots \alpha_{n}}, \mathbb{R}\right)
$$

(here $F^{0}\left(U_{\alpha 0 \ldots \alpha_{n}}, \mathbb{R}\right)$ is the vectorspace of locally constant functions $U_{\alpha_{n}} \cap \ldots \cap U_{\alpha_{n}} \rightarrow \mathbb{R}$ ), and the boundary operator $\delta: C^{n}(\mathscr{U}, \mathbb{R}) \rightarrow C^{n+1}(\mathscr{U}, \mathbb{R})$ is defined as follows: if

then

$$
f=\left\{f_{\alpha_{0} \ldots \alpha_{n}}\right\} \in C^{n}(\mathscr{U}, \mathbb{R}),
$$

$$
(\delta f)_{\alpha_{0} \ldots \alpha_{n+1}}=\sum_{i=0}^{n+1}(-1)^{i} f_{\alpha_{0} \ldots \hat{\alpha}_{i} \ldots \alpha_{n+1}} .
$$

The cohomology of this complex is called the Cech cohomology of the good cover $\mathscr{U}$, and is written as $H(\mathscr{U}, \mathbb{R})=\left\{H^{n}(\mathscr{U}, \mathbb{R})\right\}_{n}$.

De Rham's theorem for Čech cohomology is the statement that there is a canonical $\mathbb{R}$-linear isomorphism

$$
\text { (*) } H(M) \stackrel{\sim}{\rightarrow} H \cdot(\mathscr{U}, \mathbb{R}) .
$$

Consequently, $H \cdot(\mathscr{U}, \mathbb{R})$ does not depend on the good cover $\mathscr{U}$. Another immediate corollary is that since compact manifolds have finite good covers, their De Rham cohomology $\mathbb{R}$-modules are finite dimensional.

The proof of the existence of the isomorphism (*) given by A. Weil (cf. [14]) is completely constructive and explicit, and hence valid in the synthetic context. Consequently, since the embedding $s: \mathscr{M} \hookrightarrow \mathscr{G}$ preserves the ingredients of Weil's proof (notably good open covers and partitions of unity, as proved in Moerdijk and Reyes [10]), we obtain the following analogue of our first theorem of section 1.

Theonem. For any $M \in \mathscr{M}$ and any good cover $\mathscr{U}$ of $M$, there is a canonical $R$-linear isomorphism

in the topos $\mathscr{G}$.

$$
H^{q}(s M) \stackrel{\sim}{\rightarrow} H^{q}(s(\mathscr{U}), R)
$$

Turning to singular cohomology, we actually do not know whether De Rham's theorem holds in $\mathscr{G}$, at least if we interpret singular cohomology as the cohomology of the complex

$$
\ldots \rightarrow \operatorname{Hom}_{R}\left(S_{q}(M), R\right) \stackrel{\partial^{*}}{\rightarrow} \operatorname{Hom}_{R}\left(S_{q+1}(M), R\right) \rightarrow \ldots
$$


which is the dual of the complex of singular homology

$$
\ldots \rightarrow S_{q+1}(M) \stackrel{\partial}{\rightarrow} S_{q}(M) \rightarrow \ldots
$$

of Section 1. In this case, some form of the axiom of choice seems needed to establish the result. The problem here is that the dual of a short exact sequence is not necessarily exact. Explicitly,

Question. Does the singular cohomology of a manifold $s(M) \in \mathscr{G}$ coincide with its De Rham cohomology in $\mathscr{G}$, or is this version of De Rham's theorem essentially nonconstructive?

A way of circumventing this problem in the topos $\mathscr{G}$ is to replace the sheaf

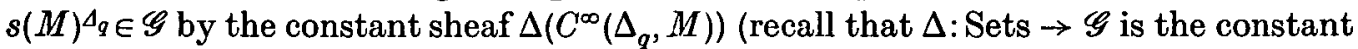
sheaf functor). We then let $S_{\Delta, q}(s M)$ be the free $R$-module in $\mathscr{G}$ generated by the constant $q$-simplices $\Delta\left(C^{\infty}\left(\Delta_{q}, M\right)\right)$ and define the corresponding cohomology $H_{\Delta}^{q}(s M)$. Exactly as before, we obtain

Theorem. Let $M \in \mathscr{M}$. Then the canonical R-linear map

is an isomorphism of the topos $\mathscr{G}$.

$$
\begin{aligned}
H^{q}(s M) & \rightarrow H_{\triangle}^{q}(s M) \\
{[\omega] } & \mapsto\left([\gamma] \mapsto \int_{\gamma} \omega\right)
\end{aligned}
$$

We should notice, however, the schizophrenic character of the isomorphism: we integrate internal (variable, in $\mathscr{G}$ ) forms $\omega$ over external (constant, from Sets) chains $\gamma$.

\section{Applications: smooth versions of De Rham's theorem}

One way of formulating De Rham's theorem 'smoothly in parameters' is to construe the De Rham cohomology groups and the (duals of the) singular homology groups as sheaves of smooth modules over the space of parameters. De Rham's theorem is then the assertion that 'fibrewise integration' induces a canonical isomorphism between the two.

In this section we derive several such versions of De Rham's theorem from the corresponding ones in $\mathscr{G}$, simply by restricting all sheaves (and natural transformations) involved on the site $\mathbb{G}$ to sheaves on the parameter space $X \in \mathscr{M}$ (Notice that the category $\mathcal{O}(X)$ of open subspaces of $X$ and inclusions is a subcategory of $\mathbb{G}$ ). The problem is a simple one of unravelling the different notions thus obtained.

We shall use script letters for the restrictions to $\mathscr{O}(X)$ of the sheaves on $\mathbb{G}$ introduced earlier; for example,

$$
\begin{aligned}
\Lambda^{q}(M) & =\Lambda^{q}(s M)_{1 O(X)} \\
\mathscr{S}_{\Delta}^{q}(M) & =S_{\Delta}^{q}(s M)_{\mid \mathcal{Q}(X)}, \quad \text { etc. }
\end{aligned}
$$

The following explicit descriptions of these sheaves on $X$ are now straightforward:

$$
\mathbb{R}_{\infty}=R_{10(X)}
$$

is the sheaf of smooth real-valued functions, i.e.

$$
\mathbb{R}_{\infty}(U)=C^{\infty}(U, \mathbb{R})
$$

for every open $U \subseteq X$, with obvious restrictions. 
$\Lambda^{q}(M)(U)=$ the set of $q$-forms on $U \times M$ which locally are of the form

$$
\sum_{i_{1}<\ldots<i_{q}} f_{i_{1}, \ldots i_{q}}(u, \mathbf{m}) d m_{i_{1}} \wedge \ldots \wedge d m_{i_{q}}
$$

(with all functions $f_{i_{1} \ldots i_{q}}$ smooth). $\Lambda^{q}(M)$ can be shown to be a sheaf, with obvious restrictions, by a standard partition of unity argument. The restriction to $\mathcal{O}(X)$ of exterior differentiation is the natural transformation

$$
\Lambda^{q}(M) \stackrel{d^{q+1}}{\longrightarrow} \Lambda^{q+1}(M), \quad d^{q+1}=\left\{d_{U}^{q+1}\right\}_{U},
$$

where the components $d_{U}$ are the usual exterior differentiation maps, but with respect to the $\mathbf{m}$-variables only. Furthermore, we have that

$$
\begin{aligned}
\mathscr{F} q(M)(U) & =\operatorname{Ker}\left(d_{U}^{q+1}\right), \\
\mathscr{E}^{q}(M)(U) & =\operatorname{Im}\left(d_{U}^{q}\right), \\
\mathscr{H} q(M)(U) & =\mathscr{F}^{q}(M)(U) / \mathscr{E}^{q}(M)(U),
\end{aligned}
$$

while a partition of unity argument again shows that $\mathscr{E}^{q}(M)$ and $\mathscr{H}^{q}(M)$ are indeed sheaves on $X$.

Turning to singular homology, we easily verify

$\mathscr{S}_{q}(M)(U)=$ set of elements which locally look like formal expressions of the form

$$
\sum_{i=1}^{n} a_{i}(u) \sigma_{i}(u, t) \text { with both } a_{i}: U \rightarrow \mathbb{R} \text { and } \sigma_{i}: U \times \Delta_{q} \rightarrow M \text { smooth. }
$$

In other words, $\mathscr{S}_{q}(M)$ is the associated sheaf of the presheaf which assigns to an open $U \subseteq X$ the free $\mathbb{R}_{\infty}(U)$-module generated by the $C^{\infty}$-maps $U \times \Delta_{q} \rightarrow M$. The restriction of the boundary operator is the obvious natural transformation

$$
\mathscr{S}_{q+1}(M) \stackrel{\partial_{q+1}}{\longrightarrow} \mathscr{S}_{q}(M), \quad \partial_{q+1}=\left\{\left(\partial_{q+1}\right)_{U}\right\}_{U} .
$$

As before, one checks that

$$
\begin{aligned}
& \mathscr{Z}_{q}(M)(U)=\operatorname{Ker}\left(\left(\partial_{q}\right)_{U}\right), \\
& \mathscr{B}_{q}(M)(U)=\operatorname{Im}\left(\left(\partial_{q+1}\right)_{U}\right), \\
& \mathscr{H}_{q}(M)(U)=\mathscr{Z}_{q}(M)(U) / \mathscr{B}_{q}(M)(U),
\end{aligned}
$$

using partitions of unity to show that these presheaves are indeed sheaves.

From the first theorem of Section 1, De Rham's theorem in $\mathscr{G}$, we now obtain immediately

Theorem (De Rham's theorem with parameters). The canonical $\mathbb{R}_{\infty}$-linear map

$$
\mathscr{H}^{q}(M) \stackrel{I}{\rightarrow} \mathscr{H}_{q}(M)^{*}
$$

of $\mathbb{R}_{\infty}$-modules on the ringed space $\left(X, \mathbb{R}_{\infty}\right)$ given by the components

is an isomorphism of sheaves.

$$
I_{U}: \mathscr{H}^{q}(M)(U) \rightarrow \mathscr{H}_{q}(M)^{*}(U), \quad I_{U}([\omega])([\gamma])=\int_{\gamma} \omega
$$

Here $\mathscr{H}_{q}(M)^{*}$ is the dual of $\mathscr{H}_{q}(M)$ in $\mathbb{R}_{\infty}$-Modules, i.e. $\mathscr{H}_{q}(M)^{*}(U)$ is the set of natural transformations from $\mathscr{H}_{q}(M)_{\mid O U(U)}$ to $\mathbb{B}_{\infty<\mid O U)}$. 
Conollary. Let $\omega$ be a smooth $X$-form on $M$, i.e. $\omega \in \Lambda^{q}(M)(X)$. If for each parameter value $x \in X$, the form $\omega(x,-) \in \Lambda^{q}(M)$ is exact, then there is a global $X$-form $\alpha \in \Lambda^{q-1}(M)(X)$ such that $\omega=d \sigma$.

Proof. The preceding theorem tells us that

$$
0 \rightarrow \mathscr{E} q(M) \rightarrow \mathscr{F} q(M) \rightarrow \mathscr{H}_{q}(M)^{*} \rightarrow 0
$$

is an exact sequence of $\mathbb{R}_{\infty}$-Modules over $X$. Since $\int_{\gamma} \omega=0$ for all $\gamma \in \mathscr{Z}_{q}(M)(X)$, $\omega$ is locally in $\mathscr{E} q(M)$, i.e. $\omega \in \mathscr{E} q(M)(X)$ since this is a sheaf.

Recently, this corollary was independently obtained by Glass [5]. Both Glass and we were unaware of the existence of an earlier proof using the method of carapaces of H. Cartan, which was pointed out to us by W. van Est (cf. [4]).

From the Comparison Theorem of Section 1 we similarly obtain

Theorem (Comparison Theorem), Let $S$ be any set, $M \in \mathscr{M}$ and let $X \in \mathscr{M}$ be the space of parameters. Then

$$
\begin{aligned}
H_{q}(M) \cong \oplus_{S} \mathbb{R} \quad \text { iff } \quad \mathscr{H}_{q}(M) \cong \oplus_{S} \mathbb{R}_{\infty}, \\
H^{q}(M) \cong \Pi_{S} \mathbb{R} \quad \text { iff } \quad \mathscr{H}^{q}(M) \cong \Pi_{S} \mathbb{R}_{\infty}
\end{aligned}
$$

(on the left-hand side $\oplus$ and $\Pi$ denote sum and product of real vector spaces; on the righthand side of $\mathbb{R}_{\infty}$-Modules in sheaves over $X$ ).

Note that, by the classical De Rham theorem, $H^{q}(M)$ is always of the form $\Pi_{S} \mathbb{R}$ (being the dual of a real vectorspace).

As a further consequence, we derive that the De Rham cohomology $\mathbb{R}_{\infty}$-Modules are vectorbundles, provided we ensure that their dimensions are finite:

Corollary. Let $T \hookrightarrow M$ be a retract of a manifold $M \in \mathscr{M}$ of finite homology type (i.e. such that $H_{q}(M)$ is finite dimensional for each $q \geqslant 0$ ). Then for each $X \in \mathscr{M}$, the $\mathbb{R}_{\infty}-$ Module $\mathscr{H}^{q}(T) \in \operatorname{Sh}(X)$ is locally free, i.e. there is an open cover $\left\{U_{\alpha}\right\}$ of $X$ such that for each $\alpha$ there is an $\mathbb{R}_{\infty} \mid U_{\alpha}$-linear isomorphism of sheaves

$$
\phi_{\alpha}: \mathscr{H}^{q}(M)_{\mid U_{a}} \rightarrow \mathbb{R}_{\infty \mid U_{a}}^{n_{a}} \text {, some } n_{\alpha} \in \mathbb{N} .
$$

Proof. $\mathscr{H}^{a}(T)$ is a retract of $\mathscr{H}^{a}(M)$, which is free and of finite type by the comparison theorem. Since $\mathbb{R}_{\infty}$ is local, the result follows from Swan's theorem (see for example Reyes [13]).

From the other versions of De Rham's theorem that hold in $\mathscr{G}$, the one for Cech cohomology and the one for the hybrid $H_{\Delta}^{q}$ cohomology, we obtain two further 'smooth in parameters' versions. We will only make the second one explicit.

As a description of the restrictions of the sheaves on $\mathbb{G}$ to $\mathcal{O}(X), X$ the parameter space, we find in this case that $\mathscr{S}_{A, q}(M)$ is the sheaf whose sections are locally of the form

$$
\sum_{i=1}^{n} a_{i}(u) \sigma_{i}(t)
$$

where $a_{i}: U \rightarrow \mathbb{R}$ and $\sigma_{i}: \Delta_{q} \rightarrow M$ are smooth maps. This gives a complex of sheaves whose dual (in the category of $\mathbb{R}_{\infty}$-Modules over $X$ ) is precisely

$$
\mathscr{S}_{\Delta, q}(M)^{*} \stackrel{\partial_{g}^{*}}{\rightarrow} \mathscr{S}_{A, q+1}(M)^{*}
$$


The cohomology $\mathscr{H}_{\Delta}^{q}(M)$ of this complex is given by

$$
\mathscr{H}_{\Delta}^{q}(M)(U)=\operatorname{Ker}\left(\left(\partial_{q}^{*}\right)_{U}\right) / \operatorname{Im}\left(\left(\partial_{q-1}^{*}\right)_{U}\right),
$$

which is indeed a sheaf on $X$ as follows again by partitions of unity. By restricting the isomorphism of the last theorem of Section 2 we obtain

Theorem. Let $M$ be a manifold, and $X \in \mathscr{M}$ be the space of parameters. Then the canonical homomorphism

$$
\mathscr{H}^{q}(M) \rightarrow \mathscr{H}_{A}^{q}(M), \quad[\omega] \mapsto\left(\left[\gamma \mapsto \int_{\gamma} \omega\right]\right)
$$

of $\mathbb{R}_{\infty}$-Modules over the ringed space $\left(X, \mathbb{R}_{\infty}\right)$ is an isomorphism.

If we unravel the definitions, we obtain a result familiar in classical differential geometry (cf. van Est[4]): for elements $\sigma \in \mathscr{S}_{4, q}(M)^{*}(T), T$ an open subspace of $X$, we have (writing $\Delta$ for the constant sheaf functor $\operatorname{Sets} \rightarrow \operatorname{Sh}(X), \Gamma$ for the global sections functor $\operatorname{Sh}(X) \rightarrow$ Sets) by the adjunction $\Delta \dashv \Gamma$, the following correspondence

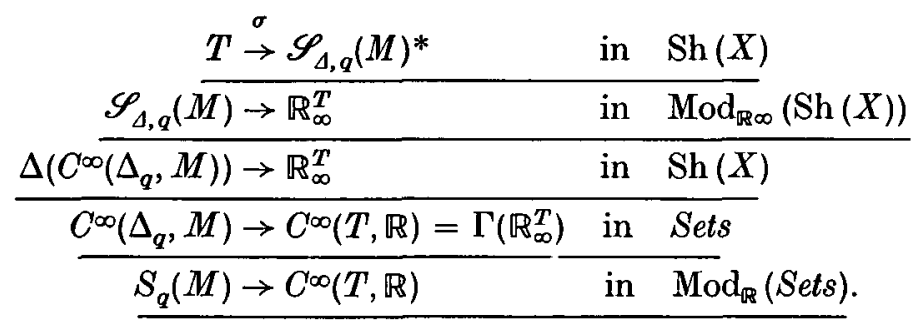

That is, a section of $\operatorname{Hom}_{\mathbb{R} \infty}\left(\mathscr{S}_{A, q}(M), \mathbb{R}_{\infty}\right)$ in $\operatorname{Sh}(X)$ over $T$ is precisely an $\mathbb{R}^{T}$-valued singular cochain in the sense of van Est [4]. Furthermore, a section of $\Lambda^{q}(M)$ over $T$ is precisely a differential $T$-form on $M$ of degree $q$ in van Est's sense. Thus, by taking the section over $T \subseteq X$ of the isomorphism of the preceding theorem, we obtain:

Corollary (van Est[4]). Let $T$ be a manifold in $\mathscr{M}$. The integration $I$ is a homomorphism of the complex $\Omega$ of $T$-forms into the complex $\Sigma$ of $\mathbb{R}^{T}$-valued singular cochains on $M$. Furthermore, the induced map

is an isomorphism.

$$
I^{*}: H \cdot(\Omega) \rightarrow H \cdot(\Sigma)
$$

Note that, as van Est points out in his paper, we can deduce the corollary of the first theorem of this section from this simpler result.

As a final remark, we note that we could have developed a 'continuous' singular homology in the topos $\mathscr{G}$, completely parallel to the (smooth) singular homology that we get when working with the manifolds $s(M) \in \mathscr{G}$ as in Sections 1 and 2: Every manifold $M$ lives in $\mathscr{G}$ not only as the smooth space $s(M)$ but also as the continuous space $c(M)$, defined as a sheaf on $\mathbb{G}$ by

$$
c(M)(\bar{A})=\operatorname{cts}(\gamma \bar{A}, M)
$$

where $\gamma \bar{A}$ is the space of points of $\bar{A}$, i.e. if $A=C^{\infty}\left(\mathbb{R}^{n}\right) / I, \gamma \bar{A}=\mathscr{Z}(I)=\left\{x \in \mathbb{R}^{n} \mid f(x)=\right.$ 0 , all $f \in I\}$. Using the same arguments as for the earlier comparison theorems, we may derive another comparison theorem for $\mathscr{G}$ :

Theorem. For any manifold $M \in \mathscr{M}$ and any set $S$,

$$
H_{q}(M, \mathbb{R}) \cong \oplus_{S} \mathbb{R} \quad \text { in Sets iff } \quad H_{q}(c M, \mathbb{R}) \cong \oplus_{S} \mathbb{R} \quad \text { in } \quad \mathscr{G}
$$


(on the right-hand side, $\mathbb{R}$ denotes the Dedekind reals in $\mathscr{G}$, i.e. the object $c(\mathbb{R})$, cf. Moerdijk and Reyes [10]).

As before, the isomorphism on the left comes from taking global sections of the isomorphism on the right.

Similarly, we obtain a version of De Rham's theorem:

Theorem (De Rham's theorem in $\mathscr{G}$, for continuous homology). For any manifold $M \in \mathscr{M}$ and any set $S$,

$$
H^{q}(s M, R)=R^{\Delta S} \quad \text { in } \quad \mathscr{G} \quad \text { iff } \quad H_{q}(c M, \mathbb{R})^{*}=\mathbb{R}^{\Delta s} \quad \text { in } \quad \mathscr{G} .
$$

Note that in the definition of $H_{q}(c M, \mathbb{R})$, the notion of 'continuous simplex' $\Delta_{q} \rightarrow c(M)$ does not cover. We take all simplices, just as with $H_{q}(s(M), R)$, and by definition of $c(M)$ these are automatically the continuous ones.

Reinterpreting this in Sh $(X), X$ a manifold, we obtain the result saying that the 'De Rham cohomology smooth in $X$-parameters' agrees with the 'singular homology continuous in parameters'.

We would like to thank W. T. van Est and D. Siersma for helpful conversations. Our greatest intellectual debt, however, is to Pierre Menard, whose example we follow (cf. [9]).

Both authors were supported by the Netherlands Organization for the Advancement of Pure Research (ZWO). The second author also acknowledges support from the National Science and Engineering Research Council of Canada, and the Ministère de l'Education du Gouvernement du Québec.

\section{REFERENCES}

[1] R. Boтt and L. W. Tu. Differential Forms in Algebraic Topology, Graduate Texts in Math no. 83 (Springer-Verlag, 1982).

[2] K.-T. ChEn. Iterated path integrals. Bull. Amer. Math. Soc. 83 (1977), 831-879.

[3] E. J. Dubuc. $C^{\infty}$-schemes. Amer. J. Math. 103 (1981), 683-690.

[4] W. T. van Est. A generalization of the Cartan-Leray spectral sequence. Indag. Math. 20 (1958), 399-413.

[5] J. P. GLASs. De Rham cohomology with free parameters. To appear.

[6] A. Kock. Synthetic Differential Geometry, L.M.S. Lectures Note series no. 51 (Cambridge University Press, 1981).

[7] A. Kock, G. E. Reyes and B. VeIT. Forms and Integrations in Synthetic Differential Geo. metry, Aarhus preprint series 1979-80, no. 31.

[8] F. W. Lawvere. Toward the description in a smooth topos of the dynamically possible motions and deformations of a continuous body. Cahiers Topologie Géom. Differentielle 21 (1980), 377-392.

[9] P. Menard. El Ingenioso Hidalgo Don Quijote De La Mancha. Unfinished manuscript, partly edited by J. L. Borges.

[10] I. MoerdiJk and G. E. Reyes. Smooth spaces versus continuous spaces in models for synthetic differential geometry. J. Pure Appl. Algebra (in the Press).

[11] I. MoerdisK and G. E. Reyes. Cohomology theories in synthetic differential geometry. In Proceedings of the Workshop on Category Theoretic Methods in Geometry, Aarhus 1983 (in the Press).

[12] N. vaN QUE and G. E. ReYes. Smooth functors and synthetic calculus. In The L. E. J. Brouwer Centenary Symposium, Studies in Logic vol. 110, ed. A. S. Troelstra and D. van Dalen (North-Holland, 1982), pp. 377-395.

[13] G. E. Reyes. Théorie des modèles et faisceaux. Adv. Math. 30 (1978), 156-170.

[14] A. Wril. Sur les théorèmes de De Rham. Comment. Math. Helv. 26 (1952), 119-145. 